\title{
Comparison of Cellular Automata and DSP Processors towards DSP Application
}

\author{
Apurba Chakraborty, Rajib Ghosh and Arnab Mitra
}

\begin{abstract}
In this paper we have emphasized on alternative uses of Cellular Automata (CA) in Digital Signal Processing (DSP). The corollary of the distinction is centered on the parallel nature for both of the processors. For digital signal processing, parallelism has been focused with reference to hardware parallelism as well as software parallelism. Major issues for DSP implementation i.e. sampling, superposition, decomposition, Fourier transformation using CA have been covered in this paper [1]. Those discussed results ensure that CA is much efficient to be used for DSP Implementation instead of DSP processors.
\end{abstract}

Keywords--- DSP, Parallelism, Hardware Parallelism, Software Parallelism, Data Parallelism, CA

\section{INTRODUCTION}

$\mathrm{D}$ IGITAL signal processing is popular since 1960s, and after the arrival of the digital computer, it is being used in a extensive range of applications. All such applications includes: multiplexing, signal encoding, and compression in telecommunication systems, sound and video belongings in the amusement industry, image compression for space exploration, radar and sonar for military use, and medical imaging (CAT and MRI) etc. Digital signal processing is typically carried out by devoted microprocessors, renowned as digital signal processors, which execute only a single unambiguous task of signal-processing.

From the architectural viewpoint, a DSP takes a signal through an input, applies a computation to the signal, and outputs the effect. The calculations applied by a DSP processor to a signal can be quite difficult, but its functionality is still quite easy: new data goes in and changed data comes out [1].

The signals that are inputted into a DSP, start and finish their lives as continuous analog waveforms. Thus before a signal can be processed by a DSP, the signal must be altered into digital format at first. A particular DSP, identified as an analog to digital converter (ADC), carries out the analog to digital conversion (A/D conversion). The counterpart to the $\mathrm{ADC}$ is the digital to analog converter (DAC). A typical A/D conversion is shown in Figure 1.

Apurba Chakraborty, Dept. of MCA, Siliguri Institute of Technology, Siliguri, WB 734009 INDIA. E-mail: cse.apu@gmail.com

Rajib Ghosh, Dept. of CSE, Adamas Institute of Technology, Barasat, WB 700126.E-mail: rajibghosh01@gmail.com

Arnab Mitra, Dept. of CSE, Adamas Institute of Technology, Barasat, WB 700126.E-mail: mitra.arnab@gmail.com
An ADC transforms an analog signal into digital by choosing sample of the signal at regular intervals and providing the results as a $1 \mathrm{D}$ array with suitable sampling frequency, which is determined by Shannon's sampling theorem. By Shannon's sampling theorem, it is essential to sample a signal at a rate greater than double the highest frequency component in the signal in order to keep all the frequency components. In supplementary words, if a signal is sampled at a frequency less than twice the highest frequency of the signal, it may be impossible for a DSP to reproduce the analog waveform from the sampled data precisely.

In digital arrangement, a signal can be passed from the ADC to other DSPs. A number of DSPs are associated in serial over a shared bus. Data is accepted along the bus from one DSP to another DSP. For example, a typical arrangement in audio processing starts with an ADC, following that would be a filter, after that a reverb, and lastly a DAC to produce the results. Such a chain is shown below in Figure 2. On the other hand, by definition, a cellular automaton (CA) consists of a lattice of cells [2], arranged in any number of dimensions (1D, 2D, 3D, etc.). Figure 3 shows the typical arrangement of 3cell Null Boundary CA.

\section{DSP vs Parallelism:}

Both perform same function i.e. receive data and process it, DSP calculations are usually complex. A DSP chain is similar to the successive generation of a CA; calculations of CA do not usually change over time. DSP can be viewed as a one-dimensional continuous CA; signal is a continuous valued array [16].

\section{CA vs Parallelism:}

In CA cells are independent entities. CA is parallel computational devices SIMD and shared memory devices, DSP can be parallel computational, support SIMD, MIMD [16]. 




Figure 1: Sample Conversion of an Analog Signal to a Digital Signal (A/D conversion)

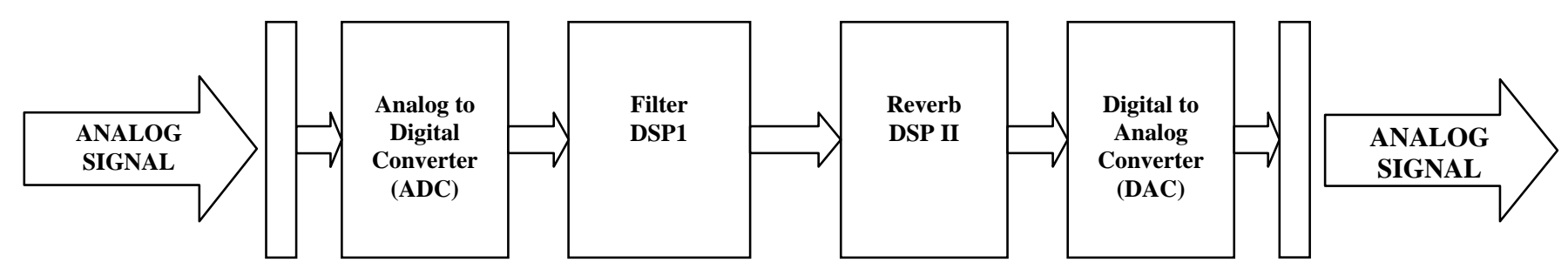

Figure 2: A typical DSP Chain Arrangement [2]

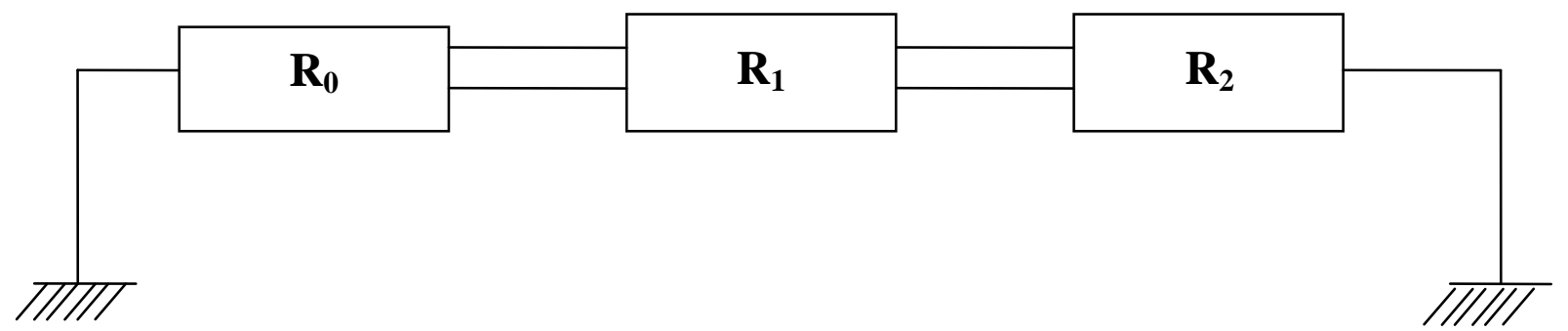

Figure 3: Typical Structure of 3-cell Null Boundary CA

Each cell contains a value. In Discrete CA, the value is function are then left behind for the next generation of cells to either 1 or 0 and in Continuous CA, the value can differ over a process. Thus a DSP is similar to cellular automata and vice specific range. Classically this range is normalized so that all values lie down between 0.0 and 1.0. Every cell in the cellular pattern performs a function on the data that is accepted to it from the earlier generation of cells. The outcomes of the versa [3], [4].

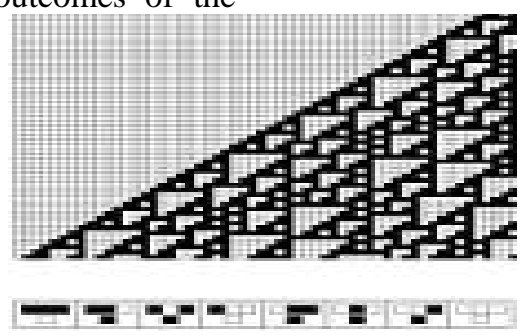

Figure 4: A 1D Discrete CA and its Equivalent Processing Rules [2]

A DSP is proposed and viewed as a cellular automaton of sorts. Both of them accept data, execute a function on the data and then produce some results. [5-8].

There are additional ways to note down the symmetries between DSPs and CAs. In a sequential chain of DSPs, every DSP is in the sequence as a different generation of cellular automata. As the data is agreed from one DSP to another, it passes down consecutive generations of computation. Once the data goes to the last DSP in the mentioned sequence, the computation is over. The main difference with this reproduction of signal with conventional CA is that every DSP in a series may achieve different computations, but in cellular automata the computation remains consistent all through each generation.

By their characteristics, cellular automata are parallel computers. CA goes from generation to generation, each cell 
performs a computation. These computations are processed sequentially. In speculative concept these computations are done at the same time instance. According to the Flynn's taxonomy, a CA would be classified as a single instruction multiple data-stream (SIMD) machine.

Parallelism is an important attribute of a CA. So if a DSP or any other schemes are compared with a CA, then it must disclose some kind of parallelism. At this point, digital signal processing is appearing as a serial job. Data is entered to the DSP system, a computation is performed on data, and final result is then as output. Every DSP accepts a signal and modifies it before sending it to the next component in that series. In early DSPs serialization was prime focus but in many modern DSPs operations are performed in parallel. As DSPs are required to process data in real time, they are extremely optimized for proficiency [9].

A "divide and conquer" approach is followed in parallel processing in order to be maximally efficient. Therefore the data that is to be processed must be parallelizable in order for a parallel system. For example, a CA's data is usually stored in a grid or an array. Each cell performs its independent computation without affecting the results of the neighboring cells.

The data signal used by DSPs is stored as a stream or an array. Therefore, it is quite parallel and well-matched for many parallel operations. In general approach for a signalsmoothing filter, the values of a data point and its adjacent neighbors are standardized. A few DSPs is capable of processing dissimilar parts of the signal without tampering the consequences of the others. There are lots of problems in digital signal processing that cannot be solved by simply changing the raw digital signal data. For these troubles we require a more convoluted way out.

In digital signal processing signals are often broken into smaller, simpler signals. This decomposition is used for some reasons. Here we sketch two of them. At first, simpler, regular signals are easier to process and most importantly, decomposition provides a means to establish an estimate of the equation that produced the signal. Acquiring a signal's equation, or an estimate thereof, is important for numerous digital signal processing functions such as encoding or decoding data on a carrier signal, frequency analysis, multiband filtering, or equalization. And as an additional benefit, decomposition produces us absolutely parallelizable data.

Each of these smaller, regular signals is processed independently after signal decomposition. The resultant signals are added together to shape the final output. Hence the final signal is a superposition of lots of smaller, simpler signals. Summing up of the decomposed and processed signals must work precisely if the functional computations performed were linear. Most digital signal processing operations are linear. A linear system has two characteristics: homogeneity and additive. The homogeneity property reveals that any alteration in the input signal's amplitude results in a resultant alteration for the output signal's amplitude. On the other hand in additive system, the sum of the resultant outputs is the similar for any two inputs as if the sum of the two inputs had been processed individually by the system.

DSP achieves parallelization in hardware: one internal and one external. The internal parallelization does not have quite radical effect on the presentation of a DSP compared to the external one.

Inside, nearly all modern microprocessors are intended with the Von Neumann architecture. A lot of DSPs, on the other hand, are projected with the Harvard architecture. The primary differentiation between these two competing architectures is that the Harvard architecture has partitioned memory banks and buses for instructions and data. Since the buses and memory storages are separate, data and instructions are retrieved in the same clock cycle.

\section{RELATED WORKS}

There are architectures that awaken upon the Harvard architecture, to be precise Analog Devices' Super Harvard Architecture, or SHARC. The key improvements SHARC designers brought about encompass an instruction cache and an I/O controller. A DSP algorithm spends for the most part of its moment in a loop, repeating operations various times over. An instruction cache retains abundant of the most recently requested instructions in registers inside the central processing unit (CPU), thereby sinking the number of requirements to the instruction memory pool and improving act. The I/O controller grants the DSP to mitigate of any $\mathrm{I} / \mathrm{O}$ operations, thus reduction of CPU cycles that would usually be washed out with $\mathrm{I} / \mathrm{O}$ requirements.

The illustration achieved by the Harvard architecture and its variants are ample, but to some degree restricted. A greater performance development may possibly be achieved by connecting numerous DSPs together to contour a multiprocessor DSP. Fortunately, this is quite talented and fairly normal with DSPs. DSPs can be agreed in both SIMD (Single Input Multiple Data stream) and MIMD (Multiple Input Single Data stream) parallel processing configurations. In the MIMD configuration, each DSP operates independently, executing diverse functions. In the SIMD design, the DSPs accomplish the accurate equal endeavor on a dissimilar separation of the signal much similar to the cells of a CA. With this approach, an absolute DSP procedure be able to be performed on the whole set of data in a single step specified a suitable number of DSPs.

When involving several processors together, DSP or otherwise, an explicit extent of exterior control logic is significant. Tasks such as directing data flow and job allotment are issued by what is well identified as "glue" logic. Unexpectedly, a lot of DSPs have this "glue" logic included and are measured for multiple processing. Most unexpected among these processors are the aforementioned SHARC processors from Analog Devices and the Texas Instruments TMS320. 
Von Neumann (Single Memory) Architecture

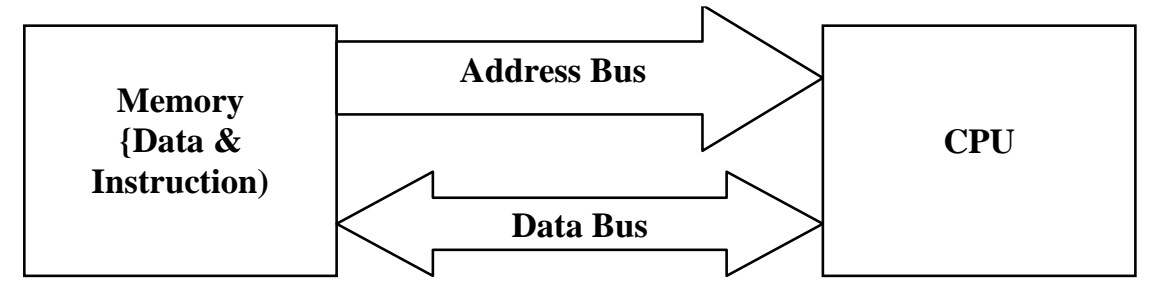

$\underline{\text { Harvard (Dual Memory) Architecture }}$

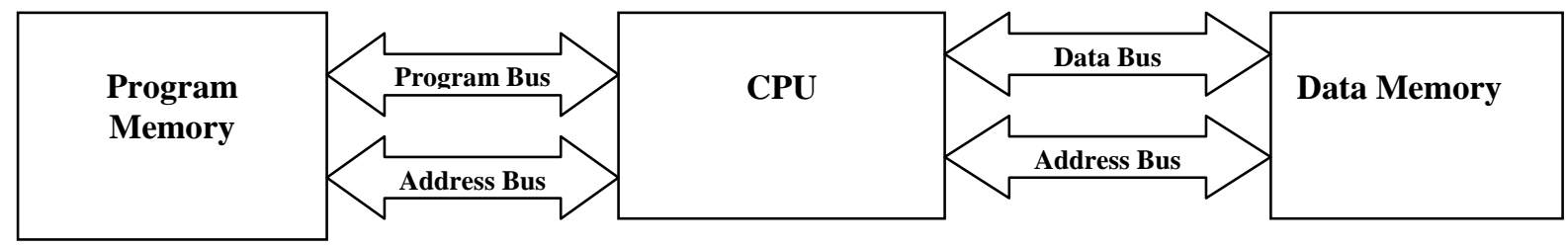

Figure 5: A Comparison of the Von Neumann and Harvard Architectures

In August 2002, IBM, Sony, and Toshiba announced that they are raising a parallel microprocessor technology. The technology, dubbed "Cell", features all over from 4 to 16 general-purpose processor cores, known as "cells", on one chip. These "cells" speak with each other via a high-speed communication bus that is well-established within the chip. Strategy utilizing a "Cell" chip can be connected together over a high-speed network to do distribute computing tasks. Sony's home video gaming console, the Play station 3, is fabricated to be the first device to relate "Cell" technology.

\section{EXPERIMENTAL RESULTS}

Study exposes that DSPs and CAs are analogous. In our approach we have used a null-boundary CA based arrangement, which has achieved the tasks like: Analog to Digital Conversion [10], Sampling [11], Superposition [12], Decomposition [13] and Fourier Transformation [14]. All these talked about tasks are being achieved by DSP using MATLAB. Our work is emphasizing on the accomplishment of same by CA, as it more cost effective solution for physical achievement of a system. Because a single CA cell just expenses for a single flip flop.

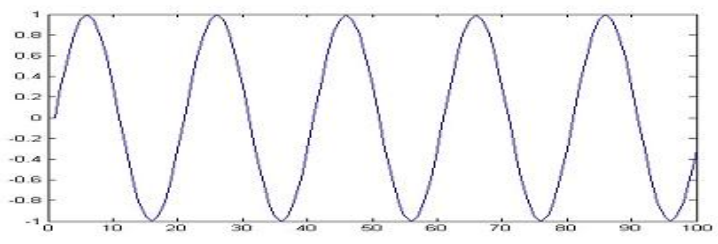

Figure 6(a): Signal Sampling of Function y1

Figure 6(a) explains a sinusoidal function with frequency $0.05 \mathrm{~Hz}$. is sampled to get 100 sampled values within the time period of 20 seconds. 


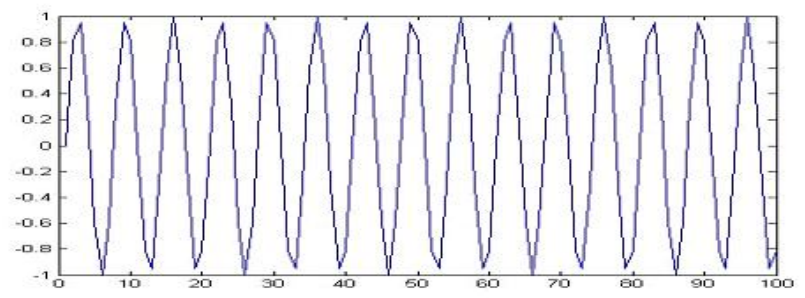

Figure 6(b): Signal Sampling of Function y2

Figure 6(b) shows a sinusoidal function with frequency $0.15 \mathrm{~Hz}$. is sampled to get 100 sampled values within the time period of 6.67 seconds.

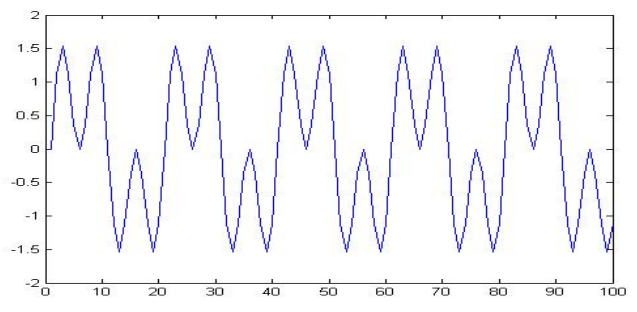

Figure 7: Sampling after the Signal Superposition

Figure 7 shows a sinusoidal function with two different frequency values $(0.05 \mathrm{~Hz}$. and $0.15 \mathrm{~Hz}$.) are sampled to get 100 sampled values within a time interval (used stem(x, y) function in MATLAB 7.0).

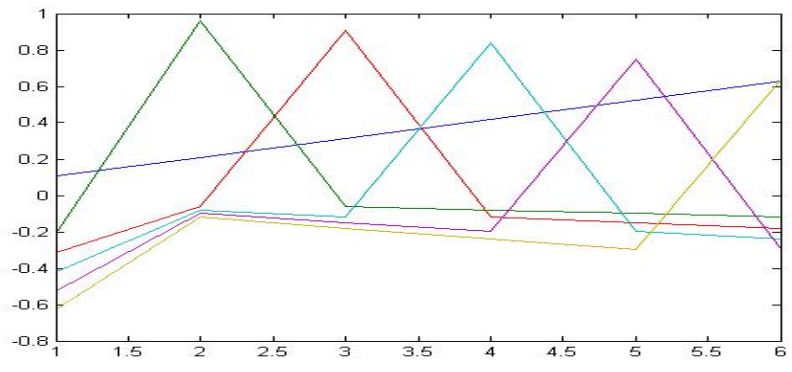

Figure 8: V-value Presentation in Signal Decomposition

Figure 8 shows a graph after plotting the different values of $\mathrm{V}$ against a sinusoidal function (svd (X)) which generate the decomposed values (U, S, V) of any signal where $\mathrm{X}$ is a set of normal input values.

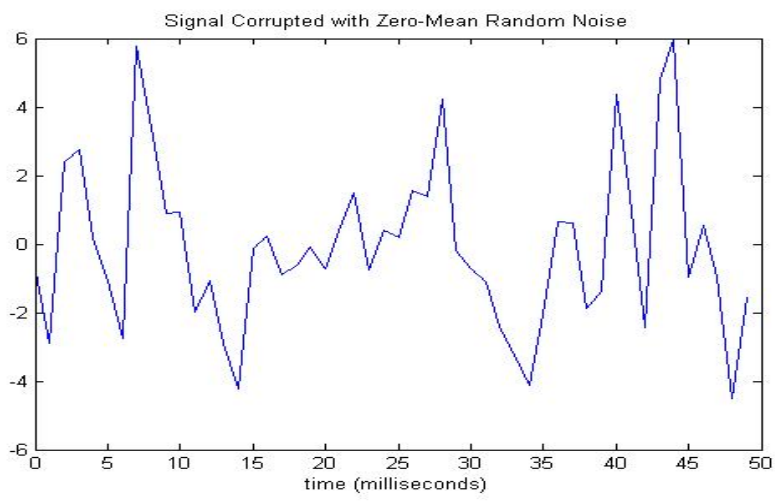

Figure 9: Fourier Transformation

Figure 9 shows the graph of a Fourier transformation where sample time, sampling frequency, length of signal, time vector has been provided to the function.

Figure 5, Figure 6, Figure 7 and Figure 8 reflects the DSP implementation in MATLAB. 


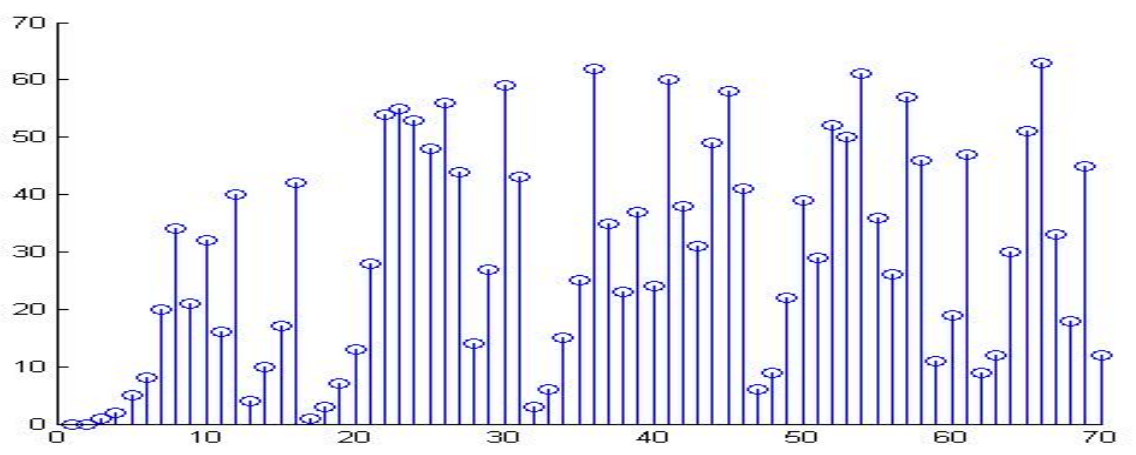

Figure 10: CA Generated Digital Signal

Figure 10 shows how a random analog input signal is converted into digital signal using 2D CA using a rule set $<90,90,90$, 90, $90,90>$.

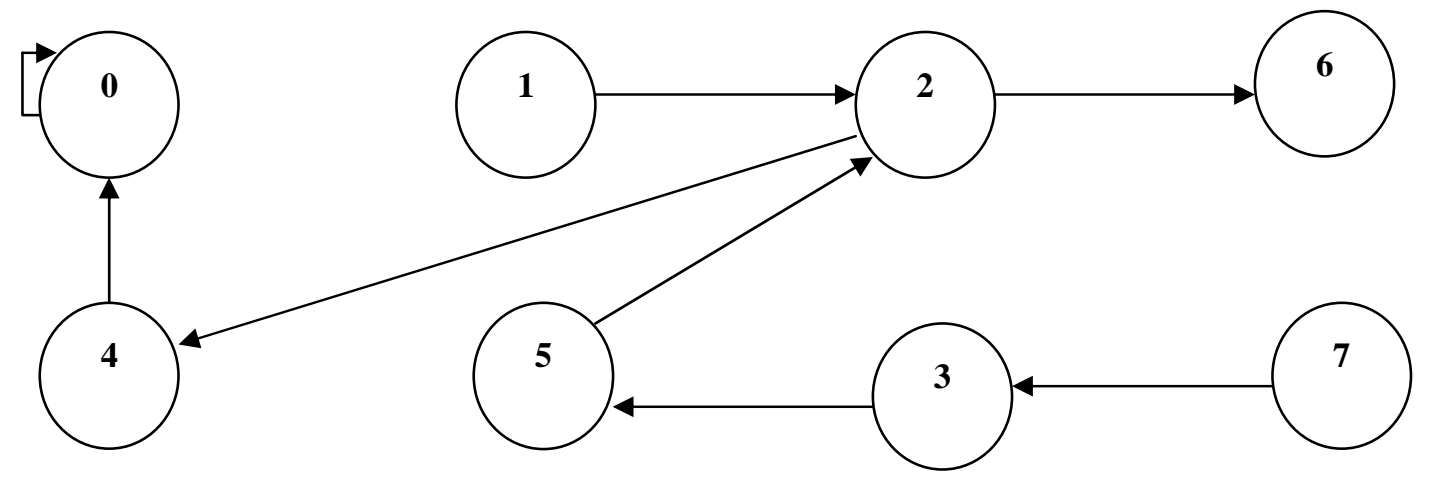

Figure 11: CA Generated Sampling Process

Figure 11 shows a CA system can sample a signal using the rule set $<226,226,226>$.
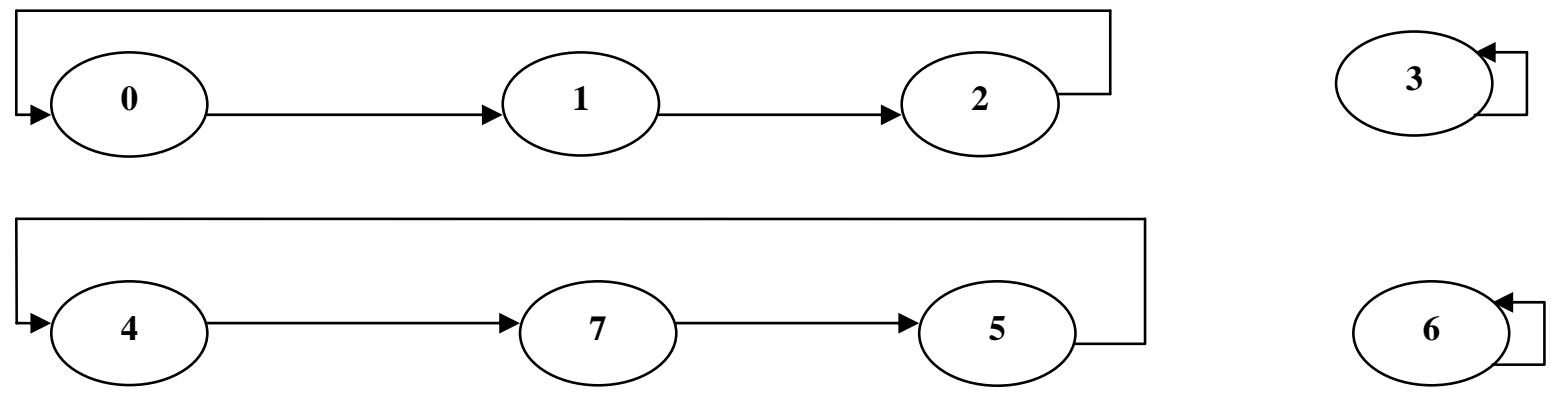

Figure 12: CA Generated Superposition of a Signal

Figure 12 shows a CA System is Performing a Superposition on Sample Signal using the Rule set <60, 90, $105>$. 


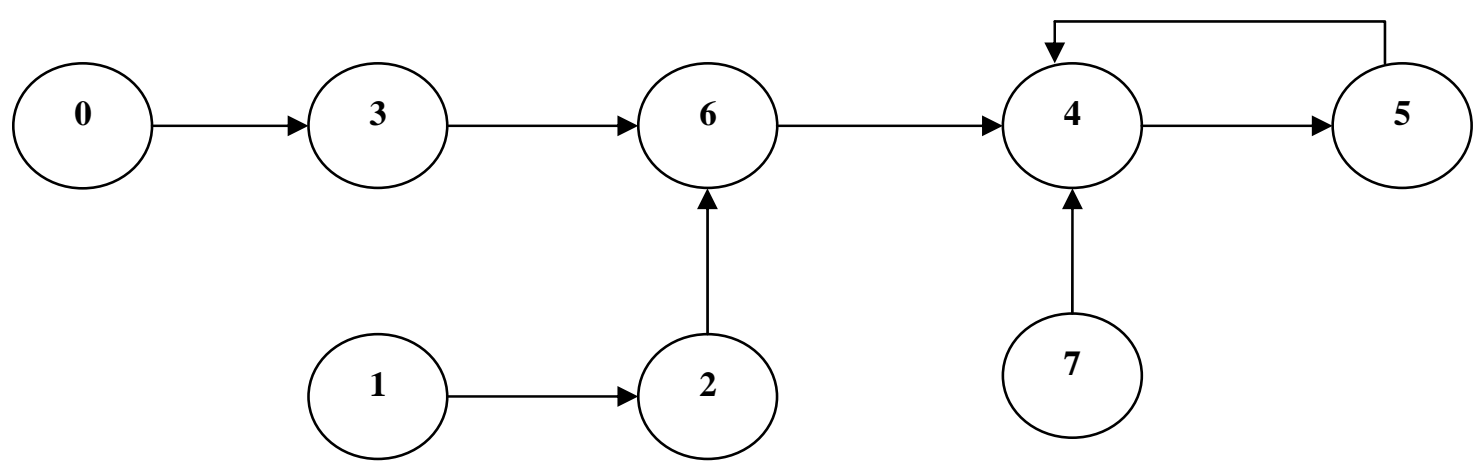

Figure 13: CA Generated Decomposition of a Signal

Figure 13 shows a CA system is performing decomposition on sample signal using the rule set $<14,15,43>$.
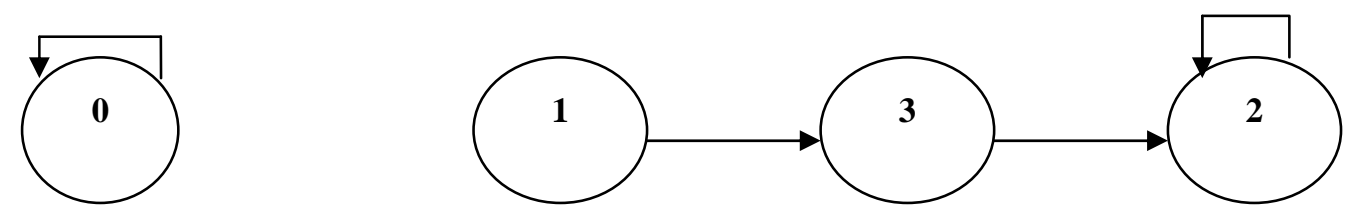

Figure 14: Fourier Transformation in CA system

Figure 14 shows a CA system is performing a Fourier Transformation using the rule set $<14,142>$. Table 1 shows the various comparison data among DSP and CA processors.

Table 1: Cost Comparison between DSP Processor and CA Processor

\begin{tabular}{|c|c|}
\hline DSP & CA \\
\hline It is application-based design. & $\begin{array}{c}\text { It is not application- } \\
\text { based design. }\end{array}$ \\
\hline $\begin{array}{c}\text { Its cost always depends on } \\
\text { FPGA/ASIC based design. }\end{array}$ & $\begin{array}{c}\text { It is D flip-flop based } \\
\text { design. }\end{array}$ \\
\hline $\begin{array}{c}\text { Huge production of DSP chip can } \\
\text { cause low cost. }\end{array}$ & $\begin{array}{c}\text { Its cost as chip as a } \\
\text { flip-flop. }\end{array}$ \\
\hline
\end{tabular}

From Table 1, comparison data among DSP and CA processors signifies that DSP processors are mainly application specific design. FPGA or ASIC based designs are available for DSP implementations. Thus the associated cost generation for a DSP processor is relatively quite high unless a bulk production is performed. On the other hand CA is a general purpose design and it is D flip-flop based design. So it is much cheaper than of application DSP fabrication cost.

Table 2 signifies the details of different rules used for CA based digital signal processing.

The mentioned Table 1 and Table 2 show about the CA implementation benefit to DSP instead of traditional DSP implementation according to its application development cost and its implementation rules. 
Table 2: Detailed Study of associated Rules

\begin{tabular}{|c|c|c|c|}
\hline Sl. No. & $\begin{array}{c}\text { CA rules in Decimal } \\
\text { Value Representation }\end{array}$ & $\begin{array}{c}\text { CA rules in Binary } \\
\text { Value } \\
\text { Representation }\end{array}$ & $\begin{array}{c}\text { Combinational Logic For } \\
\text { NextState Calculation }\end{array}$ \\
\hline 1. & 14 & 00001110 & R0 OR (R0 OR R1 OR R2) \\
\hline 2. & 15 & 00001111 & NOT R0 \\
\hline 3. & 43 & 00101011 & $\begin{array}{c}\text { R0 OR ((R0 AND R2) OR } \\
\text { (P AND (NOT Q))) }\end{array}$ \\
\hline 4. & 60 & 00111100 & R0 OR R1 \\
\hline 5. & 90 & 01011010 & R0 OR R2 \\
\hline 6. & 105 & 01101001 & R0 OR R1 OR (NOT R2) \\
\hline 7. & 142 & 10001110 & $\begin{array}{c}\text { R0 OR ((R0 OR R1) OR } \\
\text { (R0 OR R2)) }\end{array}$ \\
\hline 8. & 226 & 11100010 & $\begin{array}{c}\text { (R0 AND R1) OR (R1 } \\
\text { AND R2) OR R2 }\end{array}$ \\
\hline
\end{tabular}

\section{CONCLUSION}

Though DSPs do not make for an idyllic CA, there is a strong similarity. Most notable of these common characteristics is their use of parallelism. It is seen that, CAs are the perfect SIMD multiprocessor machine. DSP are very often SIMD. The experimental results of Figure 9-13 show an alternative solution to work with CA. This result reflects the same result as some were previously proposed.

\section{ACKNOWLEDGMENT}

We would like to thank the Bonfring International Journals to allow us to modify this template.

\section{REFERENCES}

[1] Apurba Chakraborty, Rajib Ghosh, Arnab Mitra; An Alternative Implementation of DSP Using Cellular Automata; The Second National Conference on Electronics, Communication and Signal Processing (NCECS)2012; Siliguri; Pp-92-97, 2012.

http://sjsu.rudyrucker.com/ karl.schramm/paper/

[2] S. Wolfram, A New Kind of Science. Champaign, IL: Wolfram Media, Inc., 2001

[3] Margolus N., "CAM-8: “A Computer Architecture Based on Cellular Automata". Cambridge, MA: MIT Laboratory of Computer Science. http://www.ai.mit.edu/people/nhm/cam8.pdf, 1993.

[4] E. Miranda, "CAMUS: A Cellular Automata Music Generator". http://website.lineone.net/ edandalex/camus.htm, 2002

[5] C. Rorabaugh, DSP Primer. New York: McGraw-Hill., 1998

[6] Schramm, K. "CA Sound: Sound Synthesizing 1D CA". http://sjsu.rudyrucker.com/ karl.schramm/applet/, 2003.

[7] S. Smith, (1997). "The Scientist and Engineer's Guide to Digital Signal Processing”. San Diego, CA: California Technical Publishing, 1997.

[8] J. Spooner, "Chip trio allows glimpse into Cell". CNET News.com http://news.com.com/2100-1001-948493.html?tag=fd_top, August 6, 2002.

[9] B. Wilkinson, \& M. Allen, Parallel Programming: Techniques and Applications Using Networked Workstations and Parallel Computers. Upper Saddle River, NJ: Prentice Hall, 1998.

[10] Moshe Mishali, Yonina C. Eldar, Oleg Dounaevsky, Eli Shoshan ; "Sampling: Analog to Digital at Sub-Nyquist Rates"; Cornel University Library; http://arxiv.org/abs/0912.2495

[11] Min Fang, "An instances sampling approach based on cellular automata for ensemble learning; Bio-Inspired Computing”: Theories and

Applications (BIC-TA), IEEE Fifth International Conference on, Pp- 560 564, 2010.

[12] http://www.worldscinet.com/ijmpc/22/2206/freeaccess/S0129183111016506.pdf
[13] H.V. Jagadish, T.Kailath, "Primitive $\quad$ Cellular

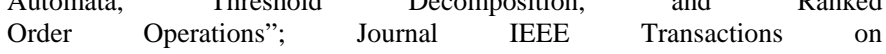
Computers; pp-148-149 doi>10.1109/12.8738, 1989.

[14] http://demonstrations.wolfram.com/download-cdfplayer.html

[15] http://sjsu.rudyrucker.com/ Karl.schramm/paper/slides.ppt 